\title{
Characterization of laser propagation through turbulent media by quantifiers based on the wavelet transform: Dynamic study
}

\author{
L. Zunino ${ }^{\mathrm{a}, *}$, D.G. Pérez ${ }^{\mathrm{b}}$, M. Garavaglia ${ }^{\mathrm{a}, \mathrm{c}}$, Osvaldo A. Rosso ${ }^{\mathrm{d}}$ \\ ${ }^{a}$ Centro de Investigaciones Ópticas (CIOp), CC. 124 Correo Central, 1900 La Plata, Argentina \\ bInstituto de Física, Pontificia Universidad Católica de Valparaiso (PUCV), 23-40025 Valparaíso, Chile \\ 'Departamento de Física, Facultad de Ciencias Exactas, Universidad Nacional de La Plata (UNLP), 1900 La Plata, Argentina \\ ${ }^{\mathrm{d}}$ Instituto de Cálculo, Facultad de Ciencias Exactas y Naturales, Universidad de Buenos Aires (UBA), Pabellón II, \\ Ciudad Universitaria, 1428 Ciudad de Buenos Aires, Argentina
}

Received 10 January 2005; received in revised form 8 August 2005

Available online 19 October 2005

\begin{abstract}
We analyze, within the wavelet theory framework, the wandering over a screen of the centroid of a laser beam after it has propagated through a time-changing laboratory-generated turbulence. Following a previous work (Fractals 12 (2004) 223) two quantifiers are used, the Hurst parameter, $H$, and the normalized total wavelet entropy. The temporal evolution of both quantifiers, obtained from the laser spot data stream, is studied and compared. This allows us to extract information on the stochastic process associated with the turbulence dynamics.
\end{abstract}

(C) 2005 Elsevier B.V. All rights reserved.

Keywords: Lightwave propagation; Turbulence; Hurst parameter; Normalized total wavelet entropy

\section{Introduction}

The purpose of this work is to statistically describe laser beam propagation through time-changing laboratory-generated turbulence. To do so, we analyze data stream corresponding to the centroid position of the laser spot by using two different quantifiers obtained from the wavelet theory: the Hurst parameter, $H$, and the normalized total wavelet entropy (NTWS). The former quantifier results from modeling the centroid's coordinates as a fractional Brownian motion $(\mathrm{fBm})$ at stationary turbulence strength [1], while the latter has been used for a wider set of stochastic processes-see Ref. [2].

The $\mathrm{fBm}$ was discovered by Kolmogorov [3] and defined by Mandelbrot and Van Ness [4] as the only family of processes which are Gaussian, self-similar, and with stationary increments. The normalized family of these gaussian processes, $B^{H}$, is the one with $B^{H}(0) \equiv 0$ almost surely, $\mathbb{E}\left[B^{H}(t)\right] \equiv 0$, and covariance

$$
\mathbb{E}\left[B^{H}(t) B^{H}(s)\right]=\frac{1}{2}\left(|t|^{2 H}+|s|^{2 H}-|t-s|^{2 H}\right),
$$

\footnotetext{
${ }^{*}$ Corresponding author.

E-mail addresses: lucianoz@ciop.unlp.edu.ar (L. Zunino), dario.perez@ucv.cl (D.G. Pérez), garavagliam@ciop.unlp.edu.ar (M. Garavaglia), oarosso@fibertel.com.ar (O.A. Rosso).
} 
for $s, t \in \mathbb{R}$. The power exponent $H$ is also known as the scaling exponent and its range is bounded between 0 and 1. The estimation of this parameter plays a key role modeling an fBm time series. One remarkable property of this family $B^{H}$ is that the $H$ parameter regulates the presence or absence of memory. In fact, it can be separated into three subfamilies accordingly: long-memory for $\frac{1}{2}<H<1$, memoryless at $H \equiv \frac{1}{2}$ (ordinary Brownian motion), and short-memory in the case $0<H<\frac{1}{2}$. Likewise, the Hurst parameter can be condensed to be the probability of the next increment of the signal having the same sign as the previous increment. Thus, it tunes the trajectory regularity. Fractional Brownian motions are continuous but non-differentiable processes (in the usual sense), and only give generalized spectra $1 / f^{\alpha}$ with exponents $\alpha$ between 1 and 3 . As a nonstationary process, the fBm does not have a spectrum defined in the usual sense; however, it is possible to define a power spectrum of the form [5]:

$$
S_{B^{H}}(f)=\frac{1}{|f|^{2 H+1}} .
$$

Remember that this equation is not a valid power spectrum in the theory of stationary processes since it is a non-integrable function in the classical sense.

Several properties evidence that wavelet analysis is well-suited to $\mathrm{fBm}$ :

(1) $\mathrm{fBm}$ is nonstationary but the wavelet coefficients form a stationary process at each scale [6,7].

(2) $\mathrm{fBm}$ exhibits a positive long-range correlation in the range $\frac{1}{2}<H<1$ but wavelet coefficients have a correlation which is highly small as soon as $N>H+\frac{1}{2}$, where $N$ is the number of vanishing moments associated with the mother wavelet $\psi(t)[7,8]$.

(3) The self-similarity of $\mathrm{fBm}$ is reproduced in its wavelet coefficients, whose variance varies as a power law as a function of scale $j[6,7]$

$$
\log _{2}\left\{\mathbb{E}\left[\left.C_{j}^{2}(k)\right|_{B^{H}}\right]\right\} \propto-(2 H+1) j .
$$

These features are due to the fact that the wavelet family $\psi_{a, b}$ is generated by dilations and translations of a unique admissible mother wavelet $\psi(t)$. So, the family itself exhibits scale invariance. It should be noted that the first two properties are valid for any process with stationary increments [9].

In particular these properties are widely used for estimating $H$ or the related spectral exponent $\alpha \equiv 2 H+1$ [5,10,11]. Through the Logscale Diagram the threefold objective: detection, identification and measurements of the scaling exponent can be achieved [12]. Basically the estimation problem turns into a linear regression slope estimation.

In order to model the time-changing turbulence situation we consider a generalization where the parameter $H$ is no longer constant, but is a continuous function of the time $t(H \rightarrow H(t))$. This generalization was introduced in financial research to model the behavior of stock market index time series [13-15]. Also, it was recently used to characterize dynamic speckle or biospeckle [16]. A single scaling exponent would be unable to show the complex dynamics inherent to the data. The constraint of stationary increments is relaxed in this case. Multifractional Brownian motion $(\mathrm{mBm})$ [17] was formalized as a class of processes which satisfies these properties.

We calculate the time-dependent Hurst exponent by using the wavelet properties. Provided that variations of $H$ are smooth enough, the signal is divided into $i$ non-overlapping temporal windows and the scaling exponent is calculated for each subset according to the procedure described in Ref. [1]. A sequence of Hurst parameter values is obtained. They give the local scaling exponent around a given instant of time. Artificially $\mathrm{mBm}$ were analyzed in order to test the quality of our estimator. In Fig. 1 one can compare the theoretical and experimental results for an $\mathrm{mBm}$ with $H$ changing linearly from 0.1 to 0.9 with $t$. The Matlab code introduced by Coeurjolly [18] was implemented to simulate the $\mathrm{mBm}$. The signal was divided into 64 temporal windows of 512 data points. We used the orthogonal cubic spline functions as the mother wavelet and the resolution levels from $j=-9$ to $j \equiv-1$.

At the same time, the NTWS is also applied to study this time-changing turbulence. Introduced as a measure of the degree of order-disorder of the signal [2], it provides information about the underlying 

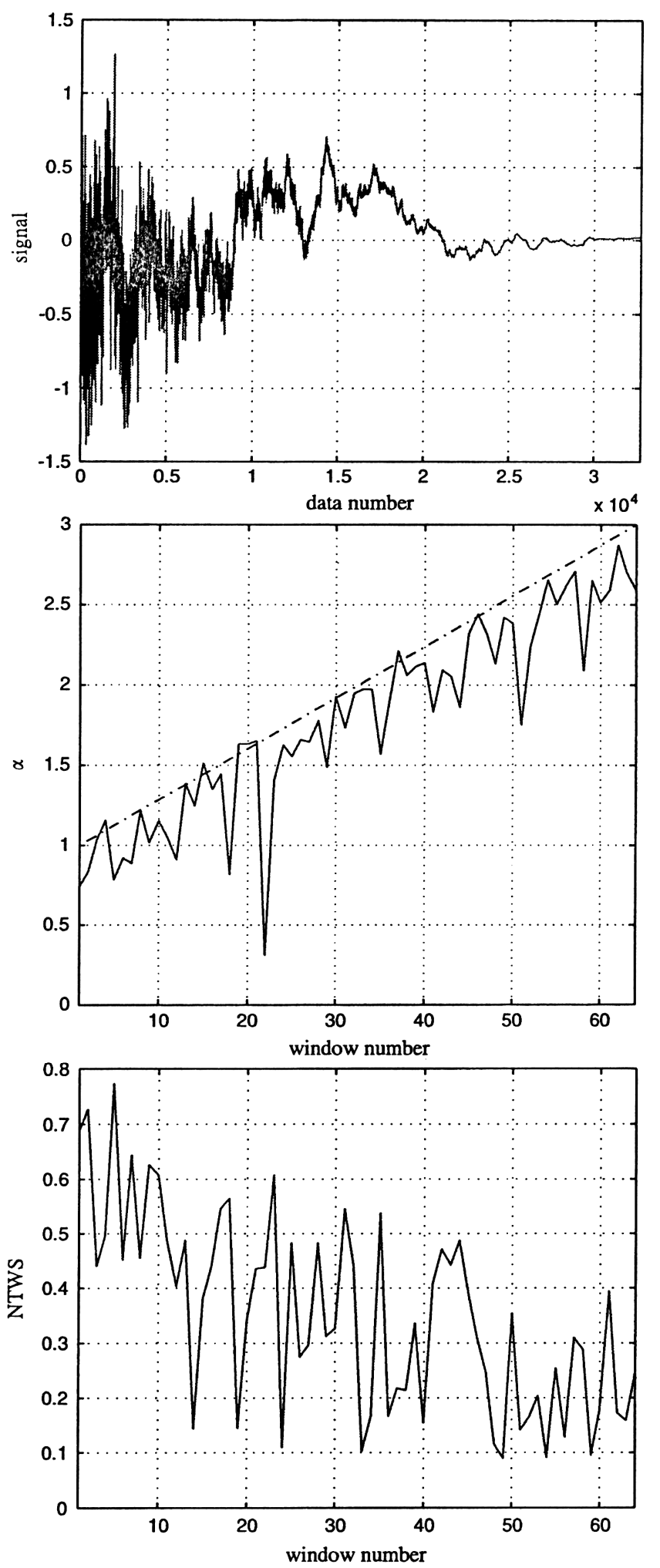

Fig. 1. Top: synthetically generated $\mathrm{mBm}$ signal with $H$ changing linearly from 0.1 to 0.9 with $t$. Middle: theoretical (dashed curve) and measured (continuous curve) Hurst parameter for this simulated mBm. Bottom: measured NTWS. 
dynamical process associated with the signal. We define the NTWS as

$$
S_{\mathrm{WT}}=-\sum_{j=-N}^{-1} p_{j} \cdot \ln p_{j} / S^{\max },
$$

where

$$
S^{\max }=\ln N
$$

with $N$ the base 2 logarithm of the number of data points and $\left\{p_{j}\right\}$ represent the relative wavelet energy (RWE). These are defined as

$$
p_{j}=\mathscr{E}_{j} / \mathscr{E}_{\text {tot }},
$$

with $\mathscr{E}_{j} \equiv \mathbb{E}\left[C_{j}^{2}(k)\right]$ being the energy at each resolution level $j=-N, \ldots,-2,-1$ and $\mathscr{E}_{t o t}=\sum_{j<0} \mathscr{E}_{j}$. They yield, at different scales, the probability distribution for the energy. It should be remarked that an orthogonal mother wavelet must be used within this theory-further details can be found in Ref. [1]. Indeed, a very ordered process can be represented by a signal with a narrow band spectrum. A wavelet representation of such a signal will be resolved in a few wavelet resolution levels, i.e., all RWE will be (almost) zero except at the wavelet resolution levels which include the representative signal frequency. For these special levels the RWE will be almost equal to one. As a consequence, the NTWS will acquire a very small, disappearing value. A signal generated by a totally random process or chaotic one can be taken as representative of a very disordered behavior. This kind of signal will have a wavelet representation with significant contributions from all frequency bands. Moreover, one could expect that all contributions will be of the same order. Consequently, the RWE will almost be equal at all resolution levels, and the NTWS will acquire its maximum possible value. Higher values for wavelet entropy means higher dynamical complexity, higher irregular behavior and, of course, lower predictability.

The time evolution of NTWS can be easily implemented. So, it is widely used to study a wide set of nonstationary natural signals. In particular, it was introduced to quantify the degree of disorder in the electroencephalographic epileptic records giving information about the underlying dynamical process in the brain [19], more specifically of the synchrony of the group cells involved in the different neural responses. Also, monthly time series of different solar activity indices (sunspot numbers, sunspot areas and flare index) were analyzed $[20,21]$. The disorder content of solar cycle activity can be derived by analyzing the wavelet entropy time evolution. Likewise, the dynamic speckle phenomenon mentioned above has also been analyzed by using these wavelet-based entropy concepts [22]. In a recent paper the relation existing between these two quantifiers - $H$ and NTWS - when they are used for analyzing $\mathrm{fBm}$ was investigated [23]. Fig. 1 (top and bottom) shows the $\mathrm{mBm}$ and its corresponding NTWS, where the same temporal windows, mother wavelet and resolution levels were used.

\section{Experimental setup and data acquisition}

The experimental measures were performed in a laboratory by producing thermal convective turbulence with two electrical heaters in a row. Three different turbulence intensities were generated by changing the amount of heat dissipated for each electrical heater: normal, soft and hard turbulence. Along the laser path three electronic thermometers sense the air temperature- $T 1, T 2$ and $T 3$; see Fig. 2 (bottom). A time series corresponding to the fluctuations of the centroid position of a laser beam's spot (wandering) over a screen, after propagation through this time-changing laboratory-generated turbulence, were recorded with a positionsensitive detector located as a screen at the end of the path. This record consists of 2,500,000 spot beam centroid coordinates' measurements with 500,000 data for each laboratory-generated turbulence condition. Further details of the experiment can be found in Ref. [1]. The temperature and signal records can be observed in Fig. 2. There, it can be observed that the turbulence is increased, and subsequently decreased to recover the initial situation. 

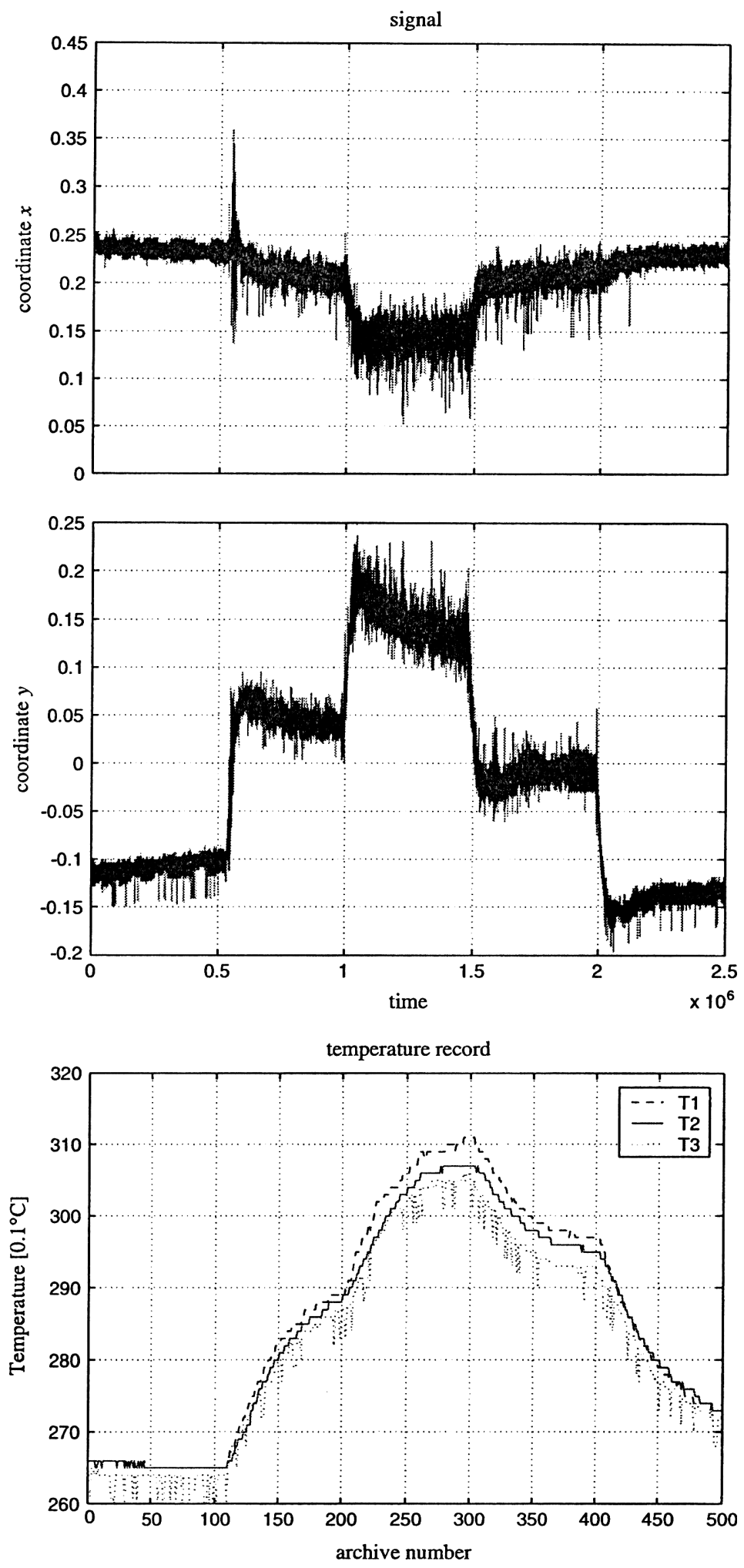

Fig. 2. Experimental records for the $x$ (top) and $y$ (middle) coordinates and the associated temperature record (bottom). 


\section{Results and conclusions}

In the present work, we employ orthogonal cubic spline functions as mother wavelets. Among several alternatives, cubic spline functions are symmetric and combine smoothness in a suitable proportion with numerical advantages. They have become a recommendable tool for representing natural signals [24,25]. The signal was divided into 606 non-overlapping temporal windows of 4096 data points. Resolution levels between $j=-7$ and $j=-3$ were used to calculate both quantifiers. The first two levels $(j=-1$ and $j=-2)$ were dropped to reduce the noise introduced by the system, while the lower levels were excluded to reduce nonstationary effects as commented in Ref. [1].

Fig. 3 shows the quantifiers' temporal evolution. Both quantifiers reveal that when the turbulence is normal the detector is not able to resolve position differences, and electronic noise associated with the detector is observed. The NTWS close to one as expected for a signal generated by a totally random process and the $\alpha$ value matches with a white noise. When the turbulence is increased the system changes in an abrupt way-see coordinates' graphs at Fig. 2. It is interesting to observe the transition between the different intensities of turbulence for the signal and its corresponding quantifiers. The Hurst parameter discriminates between the other two increased turbulences. It is possible, in average, to associate a value $\alpha_{x}=1.17, \alpha_{y}=1.04$ for the soft case and $\alpha_{x} \equiv 1.62, \alpha_{y}=1.51$ for the hard turbulence. It should be noted that the signal has more regularity for the strongest turbulence. The NTWS diminishes notably showing an increment in the order of the system but it is unable to distinguish between soft and hard turbulence giving values of $\mathrm{NTWS}_{x} \equiv 0.63, \mathrm{NTWS}_{y} \equiv$ 0.66 for the soft turbulence and $\mathrm{NTWS}_{x} \equiv 0.57, \mathrm{NTWS}_{y} \equiv 0.63$ for the hard turbulence.
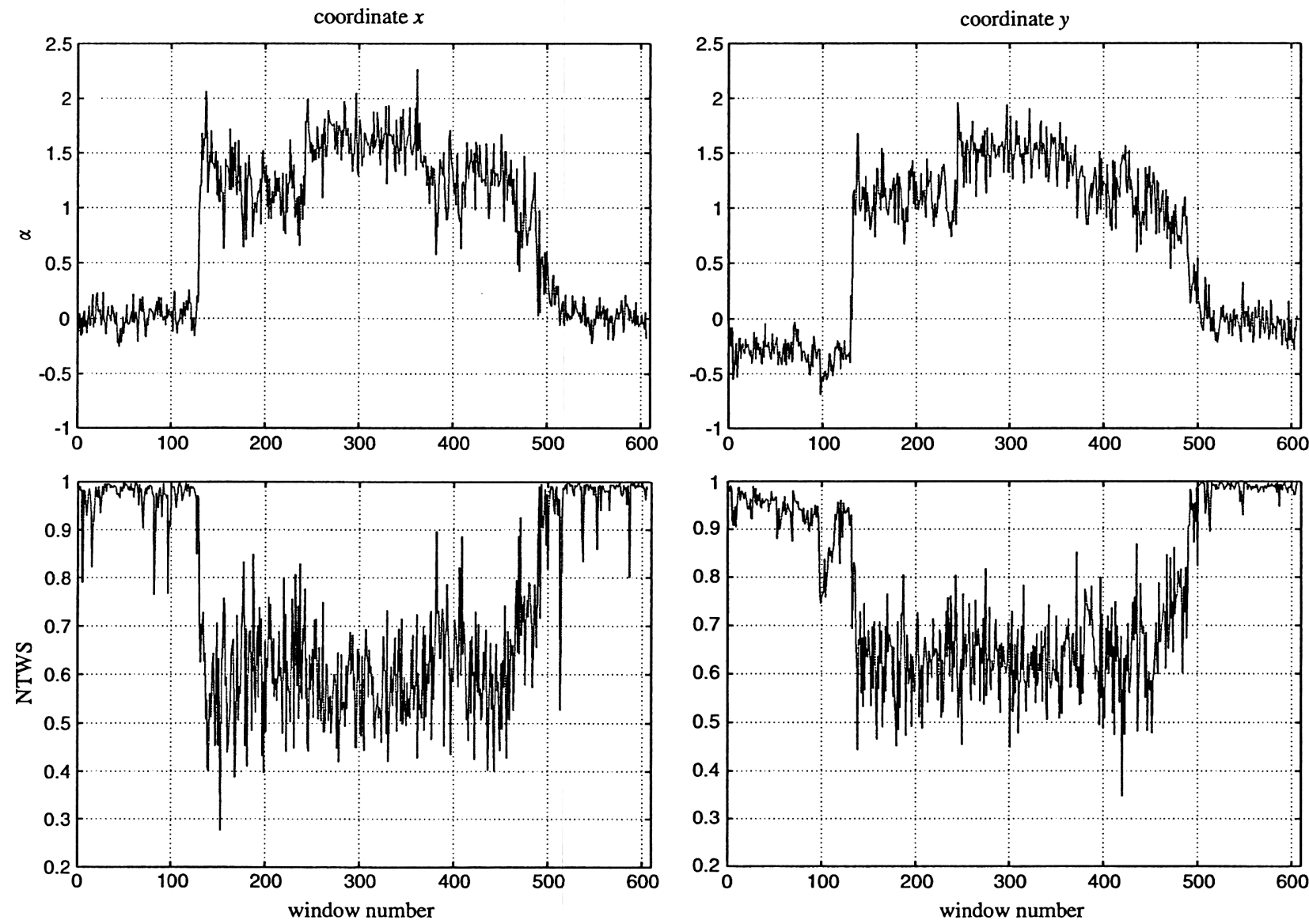

Fig. 3. Quantifiers temporal evolution: $\alpha$ (top) and NTWS (bottom) for the $x$ (left) and $y$ (right) coordinates. 
It can be followed by comparing Figs. 2 and 3 that the behavior of the signal is different for both coordinates. Nevertheless, the temporal evolution of the quantifiers is very similar. It can also be observed that the system has a hysteresis effect (see Figs. 2 and 3) as was expected.

The $\mathrm{mBm}$ model is justified for modeling the dynamics associated with these processes. We conclude that the associated scaling exponent changes continuously with the turbulence strength. In the future a new generalization will be considered and studied: the generalized multifractional Brownian motion $(\mathrm{gmBm})[26]$. These processes consider that scaling exponent variations may be very erratic and not necessarily a continuous function of time. The latter condition is a strong limitation in turbulence studies where the scaling exponent can change widely from time to time.

\section{Acknowledgements}

This work was partially supported by Consejo Nacional de Investigaciones Científicas y Técnicas (CONICET, Argentina) and Pontificia Universidad Católica de Valparaíso (Project No. 123.774/2004, PUCV, Chile).

\section{References}

[1] L. Zunino, D.G. Pérez, O.A. Rosso, M. Garavaglia, Characterization of laser propagation through turbulent media by quantifiers based on the wavelet transform, Fractals 12 (2) (2004) 223-233.

[2] O.A. Rosso, S. Blanco, J. Yordanova, V. Kolev, A. Figliola, M. Schürmann, E. Başar, Wavelet entropy: a new tool for analysis of short duration brain electrical signals, J. Neurosci. Method 105 (2001) 65-75.

[3] A.N. Kolmogorov, Wienersche Spiralen und einige andere interessante Kurven im Hilbertschen Raum, The Wiener spiral and some other interesting curves in a Hilbert space, C. R. (Dokl.) Acad. Sci. USSR 26 (1940) 115-118 (German).

[4] B.B. Mandelbrot, J.W.V. Ness, Fractional Brownian motions, fractional noises and applications, SIAM Rev. 4 (1968) 422-437.

[5] A. Peréz, C.E. D’Attellis, M. Rapacioli, G.A. Hirchoren, V. Flores, Analyzing blood cell concentration as a stochastic process, IEEE Eng. Med. Biol. (2001) 170-175.

[6] P. Flandrin, On the spectrum of fractional Brownian motions, IEEE Trans. Inf. Theory IT-35 (1) (1989) 197-199.

[7] P. Flandrin, Wavelet analysis and synthesis of fractional Brownian motion, IEEE Trans. Inf. Theory IT-38 (2) (1992) $910-917$.

[8] A.H. Tewfik, M. Kim, Correlation structure of the discrete wavelet coefficients of fractional Brownian motion, IEEE Trans. Inf. Theory 38 (2) (1992) 904-909.

[9] E. Masry, The wavelet transform of stochastic processes with stationary increments and its applications to fractional Brownian motion, IEEE Trans. Inf. Theory IT-39 (1) (1993) 260-264.

[10] P. Abry, D. Veitch, Wavelet analysis of long-range dependent traffic, IEEE Trans. Inf. Theory 44 (1) (1998) 2-15.

[11] S. Soltani, P. Simard, D. Boichu, Estimation of the self-similarity parameter using the wavelet transform, Signal Process. 84 (1) (2004) $117-123$.

[12] P. Abry, P. Flandrin, M.S. Taqqu, D. Veitch, Wavelets for the analysis, estimation, and synthesis of scaling data, in: K. Park, W. Willinger (Eds.), Self-similar Network Traffic and Performance Evaluation, Wiley, New York, 2000.

[13] A. Carbone, G. Castelli, H.E. Stanley, Time-dependent Hurst exponent in financial time series, Physica A 344 (1-2) (2004) 267-271.

[14] D.O. Cajueiro, B.M. Tabak, The Hurst exponent over time: testing the assertion that emerging markets are becoming more efficient, Physica A 336 (3-4) (2004) 521-537.

[15] D. Grech, Z. Mazur, Can one make any crash prediction in finance using the local Hurst exponent idea?, Physica A 336 (1-2) (2004) 133-145.

[16] I. Passoni, H. Rabal, C.M. Arizmendi, Characterizing dynamic speckle time series with the Hurst coefficient concept, Fractals 12 (3) (2004) 319-329.

[17] R.F. Peltier, J.L. Vehel, Multifractional Brownian motion: definition and preliminary results, Research Report RR-2645, INRIA, 1995.

[18] J.-F. Coeurjolly, Statistical inference for fractional and multifractional Brownian motions, Ph.D. Thesis, Laboratoire de Modélisation et Calcul-Institut d'Informatique el Mathématiques Appliquées de Grenoble, 2000, http://bibliotheque. imag. fr/publications/theses/2000

[19] O.A. Rosso, M.L. Mairal, Characterization of time dynamical evolution of electroencephalographic epileptic records, Physica A 312 (3-4) (2002) 469-504.

[20] S. Sello, Wavelet entropy as a measure of solar cycle complexity, Astron. Astrophys. 363 (2000) 311-315.

[21] S. Sello, Wavelet entropy and the multi-peaked structure of solar cycle maximum, New Astronomy 8 (2003) $105-117$.

[22] I. Passoni, A. Dai Pra, H. Rabal, M. Trivi, R. Arizaga, Dynamic speckle processing using wavelets based entropy, Opt. Commun. 246 (1-3) (2005) 219-228. 
[23] D.G. Pérez, L. Zunino, M. Garavaglia, O.A. Rosso, Wavelet entropy and fractional Brownian motion time series, Physica A (2005), in press.

[24] M. Unser, Spline: a perfect fit for signal and image processing, IEEE Signal Process. Mag. 16 (1999) 22-38.

[25] P. Thévenaz, T. Blu, M. Unser, Interpolation revisited, IEEE Trans. Med. Imaging 19 (7) (2000) 739-758.

[26] A. Ayache, J. Lévy Véhel, On the identification of the pointwise Hölder exponent of the generalized multifractional Brownian motion, Stochastic Processes Appl. 111 (2004) 119-156. 